\title{
DTN Based Dominating Set Routing Technique for Mobile Ad Hoc Networks*
}

\author{
Hany Samuel and Weihua Zhuang \\ Dept. of Elec. \& Comp. Eng. \\ University of Waterloo, ON, Canada N2L 3G1 \\ \{hsamuel,wzhuang\}@bbcr.uwaterloo.ca
}

\author{
Bruno Preiss \\ Research In Motion Limited \\ Waterloo, Ontario, Canada N2L $5 Z 5$ \\ bpreiss@rim.com
}

\begin{abstract}
With the wide spread of user mobility and the existence of various types of wireless networks, effective inter-networking is mandatory in order to support user roaming while maintaining the connectivity. In our previous work [1], we propose a system architecture to achieve the connectivity over a heterogeneous wireless network, which employs the delaytolerant network (DTN) concept to overcome the problem of potential intermittent connections caused by user roaming and ensures quality of service (QoS) in the presence of a long disconnection period. In this paper, we present a new routing technique for mobile ad hoc networks (MANETs) based on the DTN system architecture. We introduce the concept of virtual network topology and redefine the dominating-set based routing for the challenged network environment under consideration. In addition, we propose a time based methodology to predict the probability of future contacts between node pairs to construct the virtual network topology. Simulation results demonstrate the effectiveness of the proposed dominating-set based routing scheme under the DTN system architecture.
\end{abstract}

\section{Categories and Subject Descriptors}

C.2 [Computer-Communication Networks]: Network Architecture and Design - Wireless communication, Store and forward networks

\section{General Terms}

Algorithms, Performance, Reliability, Experimentation

\section{Keywords}

Mobile ad hoc networks, delay tolerant network (DTN), routing, quality of service (QoS), intermittent links, user mobility.

*This research was supported by research grants from the Natural Science and Engineering Research Council (NSERC) of Canada and from Research In Motion (RIM).

QShine 2008, July 28-31, 2008, Hong Kong, Hong Kong.

Copyright 2008 ICST ISBN 978-963-9799-26-4

DOI 10.4108/ICST.QSHINE2008.3931

\section{INTRODUCTION}

The recent years have seen enormous advances in wireless communication technology and co-existence of various types of wireless networks (e.g., 3rd-generation cellular networks, local area networks, sensor networks, and mesh networks). Each type of the networks is optimized for a specific networking environment, and it is impossible to have only one type of wireless networks that suits all the environments. As a result, it has become necessary to internetwork the different networks, leading to the necessity of heterogeneous wireless networking. In such an interworking scenario, how to maintain the connectivity of a mobile user poses significant technical challenges as the user roams, which can introduce an intermittent connection situation associated with challenged networks.

Challenged networks [2] can be defined as the networks where a communication path between a traffic source and its destination may never exist, or the time to send a message from the source to the destination is excessive, due to many factors such as user mobility, link failure, vertical handoff between heterogeneous networks, limited bandwidth, and path instability. The delay tolerant network (DTN) architecture has been proposed to deal with the issues in space communications. The problem of setting up a connection among nodes (e.g. spacecrafts) located deeply in space is discussed in [3]. The proposed architecture is based on an Internetindependent middleware, which is a new layer (referred to as Bundle layer). It handles the sending and receiving of the bundles (self contained messages) across the network using the underlying protocols stack and hides the nature of the communications from the upper layers (especially the application layer). The DTN concept is generalized in [2] for all the challenged networks. Due to the expected long delays, DTNs cannot accommodate any real-time applications, but mainly support time insensitive applications and the quality-of-service (QoS) provisioning is to ensure endto-end message delivery.

Routing is one of the main challenges in DTNs, where there may never exist an end-to-end physical path between a pair of traffic source and destination. Communications in DTN mainly depend on an asynchronous message forwarding mechanism, also known as store and forward mechanism. Message delivery in DTN is done through storing the message (i.e. bundle) then forwarding it either to its destination or to an intermediate node that has high probability to deliver it to the destination. For example, in the epidemic routing technique [4], each node forwards its received message to all its neighbor nodes. The message delivery mainly 


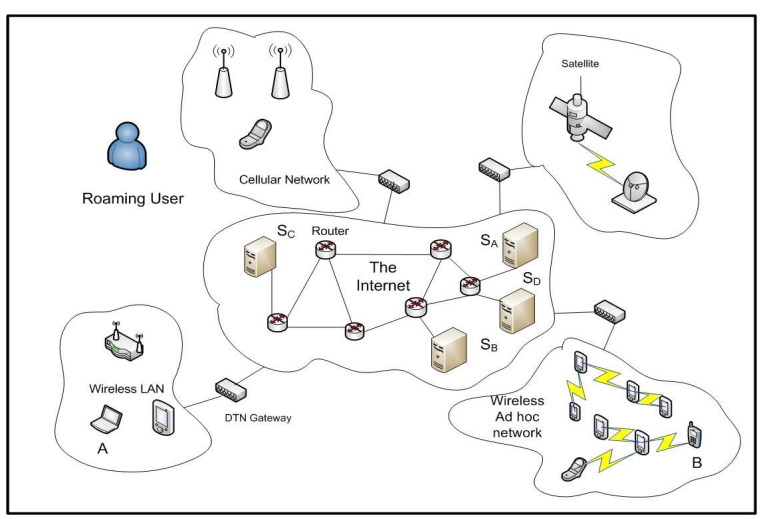

Figure 1: The system architecture with super nodes.

depends on node mobility, taking advantage that one of the message carriers may meet with the message's destination node. Routing in DTN is based on the expected nature of the contacts in the network, which can be classified as opportunistic contacts [4], scheduled contacts [5]-[6], and predicted contacts [7]-[10].

User roaming over heterogeneous wireless networks is an area that has been extensively researched (e.g., [11]-[13]). The previous solutions are mainly based on the Mobile IP protocol to deal with user mobility at the network layer. One limitation with these techniques is that, if a user is disconnected for a long period before reconnecting through another network, the current connection is terminated. Another problem is how to maintain the connection for intermittently connected users even over the same network. Based on the DTN framework, the super-node system architecture as shown in Figure 1 is proposed in [1], which provides a solution for providing a continuous connection for users that encounter these difficulties. The super-node system architecture interconnects heterogeneous wireless networks to achieve continuous connectivity for roaming users. Mobile ad hoc networks (MANETs) are considered to be an important component of the heterogeneous wireless networks for ubiquitous communications, such as in applications of emergency rescue, vehicular communications for road safety, and armored vehicle networks in a tactical environment. MANETs do not have network infrastructure support, and are therefore easy to implement. They can provide coverage over many places where mobile users may roam over and where there is no network infrastructure to provide communication services. In order to maintain a continuous connectivity for a mobile user, it is necessary that we include MANETs in the heterogeneous wireless interworking. One main problem we face is how to route messages successfully over MANETs. In [1], the epidemic routing is used for routing within MANETs, which is inefficient in terms of reliability and resources utilization. In this paper, we aim at developing a more intelligent routing technique that suits the problem domain.

There exist various routing techniques for MANETs, such as AODV [14], DSR [15] and DSDV [16]. The idea of routing in ad hoc networks based on calculating the minimum connected dominating set is introduced in [17]. The main limitation with existing routing schemes is the need for an end-to-end path between the source and the destination, which makes them unsuitable for a challenged network environment. Some research efforts are devoted to routing in a sparse ad hoc network (e.g. [18]-[20]), which depends on known routes and movements for some nodes to deliver messages. Moreover, a request may need to be sent to a moving node to change its movement trajectory to deliver the message [21]. Some routing schemes require to collect information from the moving nodes about their destination, velocity and direction of movement, which requires much computations and the awareness of destination node locations to find the best moving node to carry messages. That is, these techniques make routing decisions based on a preknown moving schedule of the mobile nodes. Such schemes are not suitable to the MANETs of interest where mobile nodes move randomly (freely) without a known schedule. The other extreme in routing is to assume no knowledge about the network, such as epidemic routing [4] which uses flooding to deliver messages and is therefore inefficient in terms of network resource usage but sometimes necessary. A compromise between the two extremes is routing based on prediction of the future movement of a node using the knowledge of its previous location and movement pattern $[7,22]$. The previous work predicts future contacts based on the number of previous contacts, which suffers from some deficiency (as discussed in section 3.2). To overcome the inadequacy, we propose here a time-based methodology for predicting the future contacts.

This paper presents a novel DTN based routing technique for challenged MANETs, with a main goal to ensure reliable message delivery with minimum cost. Our contributions are three-fold: (i) We introduce a concept of virtual network topology, which is adaptation of the network topology in a DTN context; (ii) we propose a new approach for calculating the probability of future contacts in DTN networks; and (iii) we present a routing technique that is based on calculating the dominating set for the virtual network topology, using a new algorithm for dominating set calculations. The effectiveness of the proposed routing scheme is demonstrated based on computer simulations, using a user mobility model that intends to capture the real life behavior of roaming users.

The remainder of this paper is organized as follows. Section 2 describes the system model under consideration. Section 3 introduces the concept of virtual network topology, discusses how to calculate the probability of a future contact, and proposes a new routing algorithm based on the dominating set for the virtual network topology. In Section 4 , the user mobility model is introduced and computer simulation results are presented to demonstrate the effectiveness of the proposed routing scheme. Finally, Section 5 concludes this research.

\section{THE SYSTEM MODEL}

Consider a heterogeneous wireless network, consisting of the Internet backbone and a number of wireless access networks, including cellular networks, wireless local area networks (WLANs), MANETs, sensor networks, and mesh networks. Each access network has a DTN gateway, via which it connects to the Internet. The gateways are connected over the Internet backbone, as shown in Figure 1. Associated with each mobile node is a super node located in the Internet (which can be co-located with the gateway) responsible for information delivery to the mobile node [1]. The 


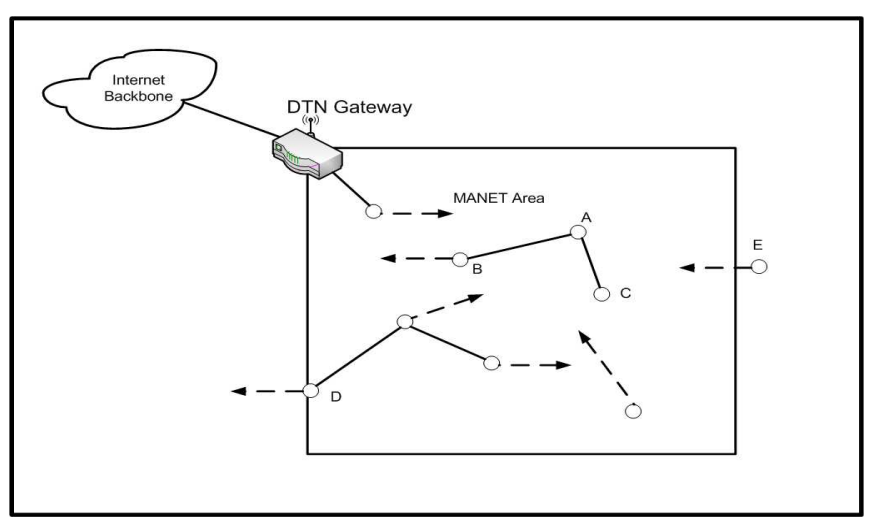

Figure 2: An illustration of the MANET under consideration.

set of super nodes are connected over the Internet backbone, and facilitate the overall information delivery from and to all the mobile nodes. We focus on routing for mobile nodes in a MANET in this paper, where the network coverage is limited by a geographical area. In the area resides a DTN gateway which connects the access network to the system. Within the MANET, there are a number of mobile nodes that can freely roam over the network coverage area. These nodes may have different communication capabilities in terms of wireless transmission range, memory size, and available transmition power. The nodes are free to enter or leave the area and consequently join or leave the network. A node can be unreliable as it can switch off at any time with or without a warning.

Figure 2 illustrates a schematic diagram for the MANET model. The DTN gateway provides connectivity to the Internet backbone. A number of nodes are roaming freely over the area covered by the network. The nodes are free to enter the area covered by the network such as node $E$ or leave the area such node $D$. Two nodes are connected when they are able to communicate with each other, i.e., when they are within each other's transmission range. For simplicity, we assume that all nodes have the same transmission range and that, if node $A$ can receive message from node $C$, node $C$ can receive from node $A$ as well. We are interested in a situation where the mobile nodes are sparsely located and the network is very likely to be partitioned, such that an end-to-end path between a pair of communicating nodes is very rare. The DTN gateway has a fixed location within the geographical area, with communication functions and capabilities similar to those of an ordinary mobile node. That is, the gateway is assumed to have a limited transmission range, and can communicate only with the nodes within its transmission range. The gateway transmission range covers only a small portion of the MANET geographical area. On the other hand, the gateway has higher processing power and larger storage (buffer) space than other roaming nodes. In terms of node mobility pattern, there is no restriction on node movements (except a reasonable upper bound on the velocity). An assumption is that some nodes usually roam toward the gateway, so that the gateway can communicate with the roaming nodes from time to time. This assumption can be satisfied by carefully choosing the gateway location, depending on the geographical features of the service coverage area.

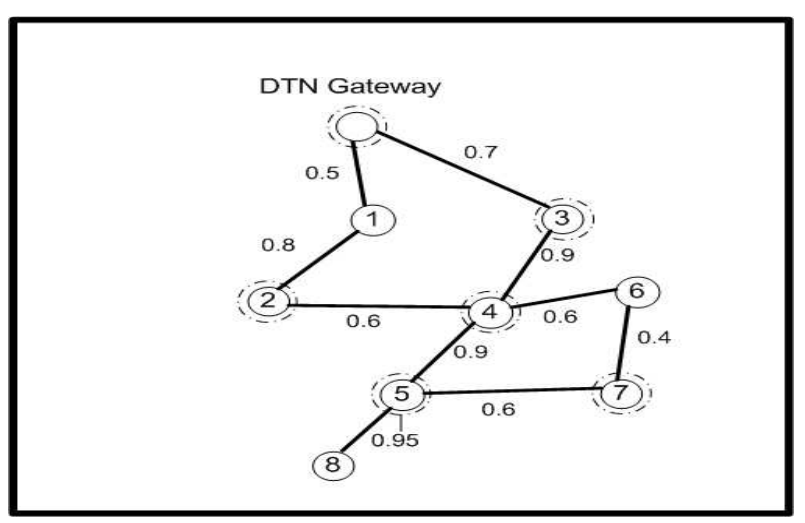

Figure 3: A simple example of virtual network topology.

\section{THE PROPOSED ROUTING SCHEME}

\subsection{Virtual Network Topology}

To route a message is to find a path from the traffic source to send this message to its destination. For the challenged network scenario that we are interested in, it is difficult, if not impossible, to find such a path. All MANET routing algorithms depend mainly on constructing the network topology and then process this topology to find a path (or paths). This technique requires that each node to have a full or partial knowledge about the network topology. Constructing and maintaining this kind of topology in our case means unnecessary overhead as the network is expected to be sparse most of the time. Furthermore, the technique fails if there is no end-to-end path between the source and destination nodes, which can happen with a high probability. Our approach to address the routing issue is to construct a virtual network topology, where a link between two mobile nodes represents the probability of future contacts (i.e. meetings) between the two nodes within the network, instead of representing the existing physical connection between the nodes. A contact is defined as the opportunity of transmitting and receiving data between two nodes as they fall within each other's transmission range. As shown in Figure 3, the network is represented as an undirected graph $G=(V, E)$, where $V$ represents the set of mobile nodes currently participating in the network and $E$ represents the set of contact probabilities for all node pairs. Note that Figure 3 illustrates only the links having a nonzero probability of future contacts.

\subsection{Probability of Future Contacts}

The main challenge in developing an efficient routing algorithm for the MANET is how to construct the virtual network topology, i.e., how to calculate the probability of future contacts between a pair of nodes. Techniques proposed for DTN routing use different criteria for predicting future contacts, e.g., the idea that a most recently met node is more probably to be met [7]. Some techniques assume that each user has a predefined movement pattern that rarely changes and the routing decisions are based on these patterns [9]. However, it is not clear how to determine the mobility patterns. Other techniques assume that the future events in the network are known in advance, which is unreasonable 


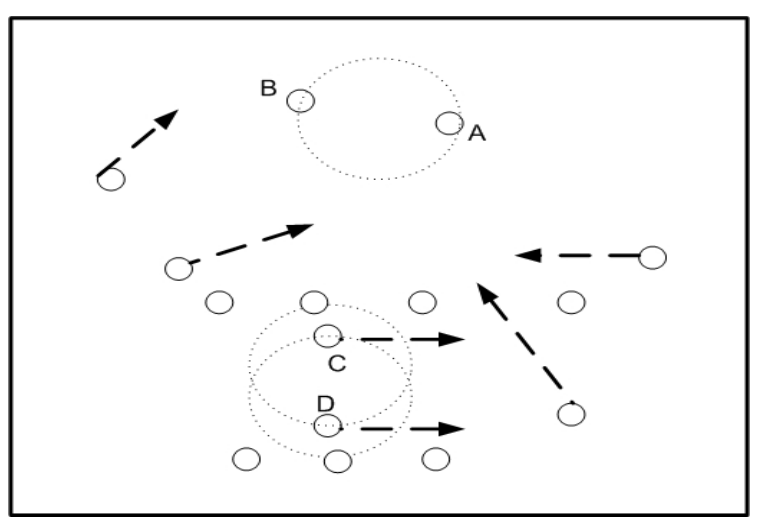

Figure 4: Problems with using the number of contacts as a parameter.

for our system where mobile users roam freely anytime and anywhere.

There is no general solution to the problem; however, the solution mainly depends on network constraints. Here, we do not assume any knowledge of the future events (e.g., node velocity, node movement direction, time instants of power on and off). Instead, we make use of network statistics that are collected and stored on the DTN gateway. The statistics are collected based on all the user sessions in the access network in the system, not only the current or most recent session. Previous techniques $[7,22]$ predict future contacts based on the number of previous contacts. Such an approach has two problems: One is multiple falsely detected contacts, as shown in Figure 4, where node $B$ is in the communication range of node $A$. As node $B$ may switch its power off and then switch it back on, node $A$ will falsely detect more than one contact with node $B$. The same situation can happen when node $B$ exhibits an intermittent connection with node $A$, e.g, due a communication barrier between them or the presence of node $B$ on the edge of node $A$ 's communication range. The other problem is related to permanent neighbors, as shown in Figure 4 where node $C$ and node $D$ move with the same velocity and in the same direction. One contact between the two nodes would be counted because no disconnection happens, independent of the long duration that the contact lasts. On the other hand, both nodes encounter other nodes as they move, which can result in multiple contacts for these nodes due to on and off links. A routing decision based on the number of contacts makes node $C$ a less suitable candidate to carry message for node $D$ than other nodes having a larger number of contacts, although node $C$ should be the favorite candidate to carry the messages as it is in continuous contact with node $D$. To address the problems, we propose routing based on the durations of previous contacts, instead of the number of previous contacts. Taking the total duration of all the contacts as the parameter is expected to give a better reflection of the likelihood that the nodes are in contact with each other. Without loss of generality, consider two nodes, $A$ and $B$. At any time, let $T_{A B}$ denote the total time that nodes $A$ and $B$ were in contact up to the moment. Regardless of time synchronization and the time durations that nodes $A$ and $B$ respectively stayed connected to the network, $T_{A B}=T_{B A}$. The probability of a future contact between nodes $A$ and $B$ is estimated approximately by

$$
P_{A B}=\frac{T_{A B}}{\left[T_{A}+T_{B}\right] / 2}
$$

where $T_{A}$ and $T_{B}$ are the total time durations that nodes $A$ and $B$ respectively connected to the network up to the moment of estimation.

Equation (1) takes into account the time durations that the nodes connected to the network. This is different from the previous techniques where the probability of meeting decreases with the time if no contact occurs, even if any or both of the nodes were not connected prior to the time of estimation. The assumption that a node is always on and connected is not practical for our problem.

Each node in the network keeps a list that contains the total duration of the meetings for each encountered node. The node sends this list to the gateway the first time it connects to the network. During the node life time in the network, it sends updates of the list to the gateway by piggyback on regular messages, or by sending special update messages if no regular messages.

\subsection{Dominating-Set Based Routing}

Our newly proposed routing scheme is based on calculating the dominating set for the virtual network topology graph. A dominating set of a graph is defined as the subset of vertices of the graph where every vertex not in the subset is adjacent to at least one vertex in the subset [17]. In our routing scheme for the MANET, the formulation of the virtual network topology and the determination of its dominating set take place at the gateway. The results are broadcast to all the mobile nodes in the network via the epidemic routing. That is, the gateway sends the information to all the nodes it encounters. These nodes, during their movements, forward the message to all other contacted nodes. The procedure continues until the information reaches all the mobile nodes in the network. On the other hand, for routing of a data packet from the source node to the destination node, the packet is forwarded only to the nodes in the dominating set, different from the epidemic routing for the message broadcasting. When a node is to send a message, it either transmits it to a node in the dominating set or to the destination node itself (if there is a direct contact). The epidemic routing based on forwarding the message to all the neighboring nodes, in anticipation that one of these nodes may meet with the destination node in the near future as it roams. On the other hand, our proposed technique counts on forwarding the message to the dominating set members only. The dominating set represents the set of nodes that have high probability to meet with all the other nodes in the network; the expected number of forwarded messages is proportional to the size of the dominating set.

To determine the dominating set, the technique given in [17] is not suitable to our virtual network topology. We should take the edge weights (i.e., the probabilities of future contacts) into consideration, as we may have a fully connected graph where most of the edges have a very low weight. Our procedure for formulating the dominating set contains two phases. In the first phase, for each node not already in the set, we add the node that it is most probable to meet to the dominating set. We process the nodes in ascending order of their ID. The second phase ensures that the dominating set is connected, which is necessary for proper forwarding of a message to the destination. As the gateway 
connects the MANET to the overall system, it should always be included in the dominating set. Algorithm 1 shows the details of our proposed algorithm, where $D S$ represents the dominating-set and $N(i)$ represents the set of neighbors for node $i$. As an example to explain the algorithm, con-

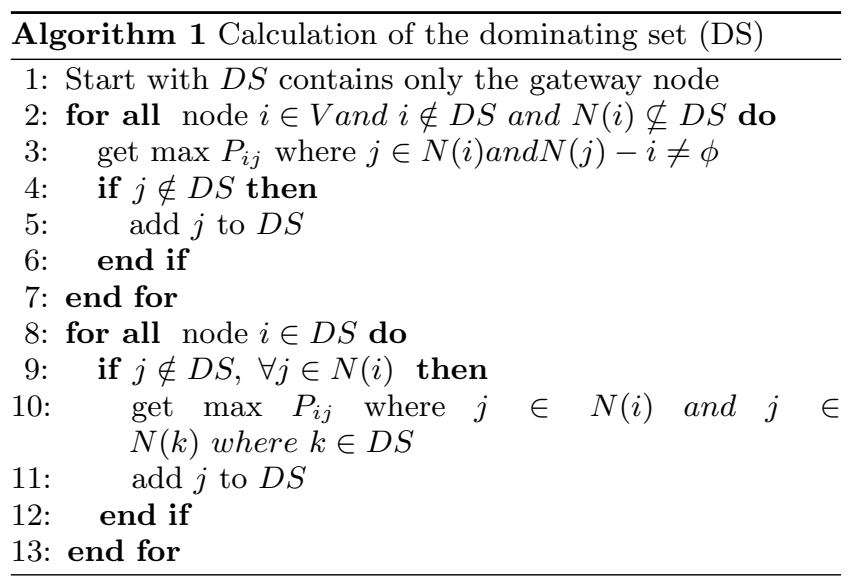

sider the simple virtual network topology in Figure 3. After constructing the virtual network topology based on the probability information of future contacts, the procedure to determine the dominating set starts as follows. First, we start with the $D S$ containing only the gateway node. Processing node $A$ adds node $B$ to the $D S$. As node $B$ is now an element of $D S$, it is not processed. Processing node $C$ adds node $D$ to the $D S$. Processing node $E$ and node $F$ would add node $D$ which is already in the $D S$. Processing node $G$ adds node $E$ to the $D S$. Note that node $H$ will not be processed as all its neighbors are already in the $D S$. After the first phase, $D S=\{$ gateway, node $B$, node $D$, node $E$ \}. The second phase finds that the $D S$ is not connected as the gateway has no neighbor in the $D S$, and therefore adds node $C$ to the dominating set. Note that the proposed algorithm may not result in the minimum dominating set. Minimizing the dominating set is a topic for further research.

\section{PERFORMANCE EVALUATION}

\subsection{The Node Mobility Model}

The proposed calculation of the probability of future contacts is based on the observations prior to the estimation instant. As in real life users usually follows specific patterns in their movements, we consider the following user mobility model. The geographical area covered by the MANET is partitioned to $m$ partitions. When a node is connected to the network, it visits each of the partitions with a certain probability. The location of a mobile node in the future is independent of its location in the past, given its current location. Denote the location state of a mobile node by the partition it resides, and assume the residence times of all the mobile nodes in each partition are iid exponential random variables. Then, the user mobility model can be characterized by a one-dimensional continuous-time Markov chain, with location state space $\{1,2, \ldots, m\}$, as shown in Figure 5 . The user movement model over the network coverage area is described by the transition matrix $M$ of the Markov

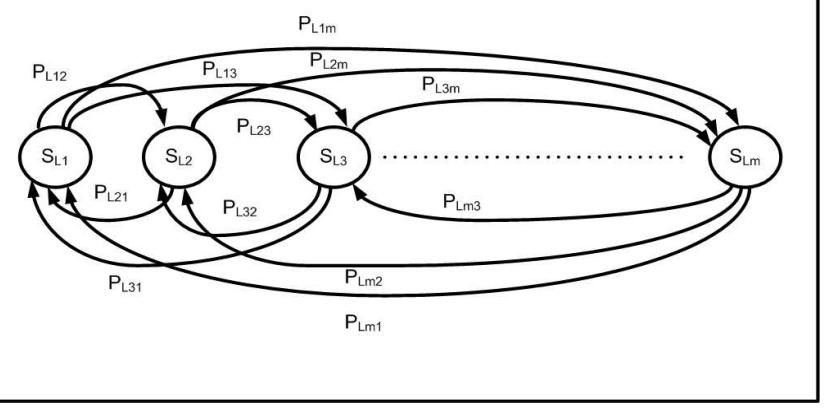

Figure 5: Modeling of user movement by a finitestate Markov chain.

chain, given by

$$
\mathbf{M}=\left(\begin{array}{cccc}
P_{L_{11}} & P_{L_{12}} & \ldots & P_{L_{1 m}} \\
P_{L_{21}} & P_{L_{22}} & \ldots & P_{L_{2 m}} \\
\ldots & \ldots & \ldots & \ldots \\
P_{L_{m 1}} & P_{L_{m 2}} & \ldots & P_{L_{m m}}
\end{array}\right)
$$

where $P_{L_{i j}}$ is the conditional probability that a mobile node will enter partition $L_{j}$ given that it is still connected to the network and it leaves its current partition $L_{i}$. For any partition $L_{i}$, we have $\sum_{j} P_{L_{i} j}=1$. The transition probability matrix depends on the geographical characteristics of the service area and the network environment under study.

\subsection{Simulation Results}

We compare the performance of the proposed dominatingset based routing technique with that of the epidemic routing. The performance is measured in terms of the number of forwarded messages over the network to capture how efficiently each technique uses the available resources (such as radio bandwidth), and the number of undelivered messages to indicate how successful the technique is in delivering messages (a QoS measure).

In our experiments, we consider that the MANET coverage area is a rectangle of size $1500 \mathrm{~m} \times 1500 \mathrm{~m}$. The area is partitioned into $150 \mathrm{~m} \times 150 \mathrm{~m}$ partitions. Each simulation proceeds in discrete time steps. Mobile nodes have mobility trajectories independent of each other. We experiment with 50 nodes. For each simulation run, a transition matrix $M$ is randomly generated and stays fixed till the end of the simulation. Initially, the node locations are uniformly distributed over the service area. As the simulation time increases, each node (if connected) moves randomly according to the transition matrix. When a node moves to a new partition, it stays there for a residence time that is an exponential random variable with an average of 20 simulation steps. At the end of the residence time, the node will move to a new partition with a probability of 0.7 , or will disconnect from the network with a probability of 0.3 . If the node disconnects, it will stay disconnected for a duration that is exponentially distributed with an average of 20 time steps. For simplicity, we assume that a node is able to communicate only with other nodes in the same partition. Messages are generated based on a Poisson process with mean rate of $\frac{10}{3}$ messages per time step. The source and destination mobile nodes for each message are selected at random. All the messages are equal in size, with the same message live time of 40 simula- 


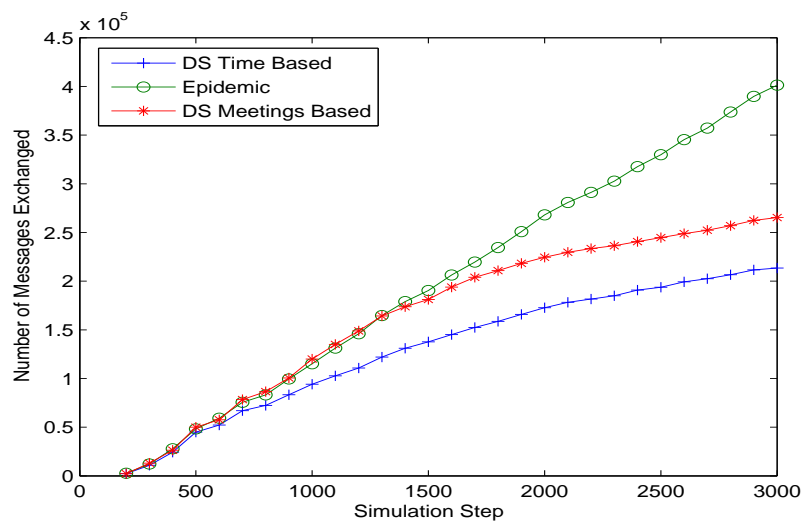

Figure 6: Comparison between the Epidemic routing and Dominating set based routing with respect to the number of messages exchanged

tion steps. The buffer space is 15 messages at each mobile node and 2000 messages at the gateway. When the node buffer is full and a new message is received, the oldest message in the buffer is removed to receive the new message. At each time step, the node detects its neighbor nodes and exchanges the buffered messages with them (the messages they do not already have) based on the routing technique used. Each node also updates its buffer by removing the expired messages. For each experiment, a communication scenario (i.e., set of messages, user connections, user disconnections, user movements) is set up randomly and run for each routing technique. Figure 6 shows a comparison between the epidemic routing technique and the dominating-set based routing technique in terms of the total number of forwarded messages. It includes the results for the dominating-set routing technique based on two different ways of calculating the probability of future contacts. One is based on the number of previous contacts and the other is based on the total contact time as discussed in Section 3.2. It is clear that the dominating-set based technique is much better than the epidemic routing in terms of the number of forwarded messages, because in the former scheme each message is forwarded only to the dominating set members, but not all the neighboring nodes as in the latter scheme. It is also observed from the figure that the time based calculation for the dominating set gives better performance than the calculation based on the number of previous contacts. On the other hand, Figure 7 demonstrates that the dominating-set based routing leads to more lost messages than the epidemic routing. A message is lost if it has not been delivered to the destination node before the message live time expires. In the new scheme, a message is forwarded only from the dominating set members to the destination, which is more likely to cause a longer delay for the destination node to meet one of the dominating set members as compared with the epidemic routing. However, when the message live time is increased to 80 time steps, the two routing schemes give comparable performance as shown in Figure 8. Figure7 also shows that calculating the probability of future contacts based on the previous contact durations leads to better routing performance (in terms of the number of undelivered messages) than calculating it based on the number of previous contacts, as the time based probability calculation results in a more accurate virtual network topology. We have carried out extensive simulations to evaluate

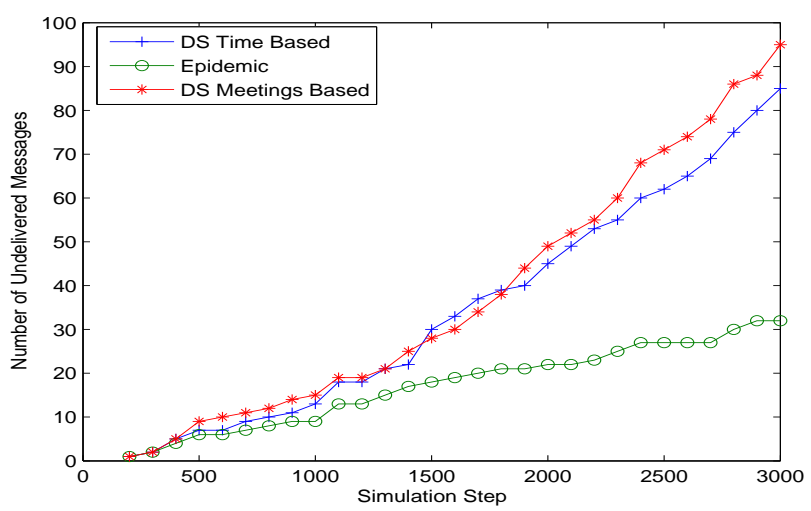

Figure 7: Comparison between the epidemic routing and dominating-set based routing with respect to the number of undelivered messages.

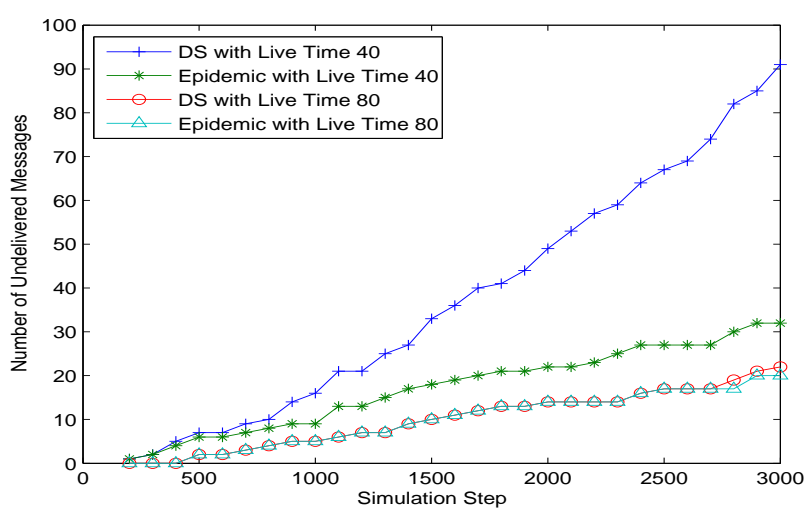

Figure 8: Comparison between the epidemic routing and dominating-set based routing with different message live times.

the performance of the proposed routing scheme. Due to space limitation, we cannot discuss all the results in detail, but summarize our observations in the following: (a) With an increasing number of mobile nodes, the dominating-set based routing gives much better performance than the epidemic routing in terms of resources utilization (measured as the number of forwarded messages), and comparable performance in terms of the percentage of undelivered messages; (b) The size of the dominating set reduces with time. As the MANET operation time increases, there are more observation data for estimating the probability of future contacts, resulting in a more accurate virtual network topology; (c) The number of forwarded messages reduces as the size of the dominating set decreases; (d) The performance of both routing schemes degrade when the buffer size is reduced, but the dominating-set based routing still outperforms the epidemic routing. Reducing the buffer size not only increases the number of undelivered messages (as expected), but also increases the number of forwarded messages due to a larger number of message retransmissions after the messages were dropped because of the buffer space limitation. 


\section{CONCLUSIONS AND FUTURE WORK}

In this paper, we introduce the concept of virtual network topology to adequately model a DTN based network with intermittent links. Constructing this topology requires a methodology to estimate the probability of future contacts between nodes in the network. We propose to determine the estimate for MANETs based on the duration of previous contacts. We then propose a new routing technique based on the dominating-set approach over the virtual network topology. A calculation of the dominating set is discussed for the virtual network topology graph. Simulation results demonstrate that the newly proposed routing scheme outperforms the epidemic routing in both resource utilization and quality of service provisioning, and that the estimation of the probability of future contacts between two nodes based on previous contact durations yields better routing performance than the estimation based on the number of contacts.

Further work on the proposed routing scheme includes generalizing the technique for all MANETs by distributed calculations of the dominating set rather than centralized calculation in the DTN gateway, which will also improve the system scalability. Calculating the dominating set using our greedy algorithm does not guarantee a minimum dominating set. How to determine an optimal dominating set is a very important issue that needs more research. We have improved the calculation of the probability of future contacts between two nodes. As the probability plays an important role in the routing performance, more research efforts should be made to study how user mobility is related to the probability and how to further improve the accuracy in the probability estimation.

\section{REFERENCES}

[1] H. Samuel, W. Zhuang, and B. Preiss, "Routing over interconnected heterogeneous wireless networks with intermittent connections," in Proc. IEEE ICC'08, May 2008.

[2] K. Fall, "A delay-tolerant network architecture for challenged internets," in Proc. ACM SIGCOMM '03, pp. 27-34, 2003.

[3] S. Burleigh, A. Hooke, L. Torgerson, K. Fall, V. Cerf, B. Durst, K. Scott, and H. Weiss, "Delay-tolerant networking: an approach to interplanetary internet," IEEE Communications Magazine, 41(6):128-136, June 2003.

[4] A. Vahdat and D. Becker, Epidemic routing for partially connected ad hoc networks. Technical Report CS-200006, Duke University, April 2000.

[5] S. Jain, K. Fall, and R. Patra, "Routing in a delay tolerant network," in Proc. ACM SIGCOMM '04, pp. 145-158, 2004.

[6] I. F. Akyildiz, Özgür B. Akan, C. Chen, J. Fang, and W. Su, "Interplanetary internet: state-of-the-art and research challenges," Computer. Networks, 43(2):75-112, 2003.

[7] A. Lindgren, A. Doria, and O. Schelén, "Probabilistic routing in intermittently connected networks," SIGMOBILE Mobile Computing and Communications Review, 7(3):19-20, 2003.

[8] X. Chen and A. L. Murphy, "Enabling disconnected transitive communication in mobile ad hoc networks," in Proc. Workshop on Principles of Mobile Computing, pp. 21-23, colocated with PODCŠ01, 2001.

[9] J. Ghosh, H. Q. Ngo, and C. Qiao, "Mobility profile based routing within intermittently connected mobile ad hoc networks (ICMAN)," in Proc. ACM IWCMC '06, pp. 551-556, 2006.

[10] J. Leguay, T. Friedman, and V. Conan, "DTN routing in a mobility pattern space," in Proc. ACM 2005 ACM SIGCOMM workshop on Delay-tolerant networking (WDTN '05), pp. 276-283, 2005.

[11] M. Shi, H. Rutagemwa, X. Shen, J. Mark, and A. Saleh, "A service-agent-based roaming architecture for WLAN/Cellular integrated networks," IEEE Trans. Vehicular Technology, 56(5):3168-3181, Sept. 2007.

[12] H. Rutagemwa, S. Pack, X. Shen, and J. Mark, "Robust cross-layer design of wireless profiled TCP mobile receiver for vertical handover," IEEE Trans. Vehicular Technology, 56(6):3899-3911, Nov. 2007.

[13] C. Guo, Z. Guo, Q. Zhang, and W. Zhu. "A seamless and proactive end-to-end mobility solution for roaming across heterogeneous wireless networks," IEEE J. Selected Areas in Communications, 22:834-848, June 2004.

[14] C. E. Perkins and E. M. Royer, "Ad-hoc on-demand distance vector routing," in Proc. 2nd IEEE Workshop on Mobile Computer Systems and Applications (WMCSA '99), pp.90-100, 1999.

[15] D. B. Johnson and D. A. Maltz, "Dynamic source routing in ad hoc wireless networks," Mobile Computing, vol. 353, pp. 153-181, Kluwer Academic Publishers, 1996.

[16] C. E. Perkins and P. Bhagwat, "Highly dynamic destination-sequenced distance-vector routing (DSDV) for mobile computers," in Proc. ACM SIGCOMM '94, pp. 234-244, 1994.

[17] J. Wu, M. Gao, and I. Stojmenovic, "On calculating power-aware connected dominating sets for efficient routing in ad hoc wireless networks," Proc. Int. Conf. Parallel Processing, pp. 346-354, Sept. 2001.

[18] R. Shah, S. Roy, S. Jain, and W. Brunette, "Data mules: modeling a three-tier architecture for sparse sensor networks," in Proc. 1st IEEE Int. Workshop on Sensor Network Protocols and Applications, pp. 30-41, 2003.

[19] W. Zhao and M. H. Ammar, "Message ferrying: proactive routing in highly-partitioned wireless ad hoc networks," in Proc. 9th IEEE Workshop on Future Trends of Distributed Computing Systems (FTDCS'03), pp. 308-314, 2003.

[20] W. Zhao, M. Ammar, and E. Zegura, "A message ferrying approach for data delivery in sparse mobile ad hoc networks," in Proc. ACM MobiHoc '04, pp. 187-198, 2004.

[21] Q. Li and D. Rus, "Sending messages to mobile users in disconnected ad-hoc wireless networks," in Proc. ACM MobiCom '00, pp. 44-55, 2000.

[22] J. A. Davis, A. H. Fagg, and B. N. Levine, "Wearable computers as packet transport mechanisms in highly-partitioned ad-hoc networks," in Proc. 5th IEEE Int. Symp. Wearable Computers (ISWC '01), pp. 141-148, 2001. 